\title{
TUNNELLING UNDER A HERITAGE STRUCTURE: DISTRIBUTED SENSING DATA AND CRACKED EQUIVALENT BEAM MODELS
}

\author{
S. Acikgoz ${ }^{1 *}$, A. Franza ${ }^{2}$, M.J. DeJong ${ }^{3}$ and R.J. Mair ${ }^{4}$ \\ ${ }^{1}$ Department of Engineering Science, University of Oxford, Oxford, UK \\ ${ }^{2}$ Department of Ground Engineering, Technical University of Madrid, Madrid, Spain \\ ${ }^{3}$ Department of Civil and Environmental Engineering, University of Berkeley. California, US \\ ${ }^{4}$ Department of Engineering, University of Cambridge, Cambridge, UK \\ * Corresponding author
}

\begin{abstract}
This paper describes the insights gathered from the application of two new sensing and analysis techniques to investigate the structural response of a Grade 1 listed masonry church undergoing tunnelling-induced movements. The church façades feature a high proportion of window openings and exhibit pre-existing damage due to past settlements. To understand the influence of tunnelling on these façades, comprehensive strain and displacement measurements were obtained. In particular, Fibre Bragg Grating (FBG) sensor chains and laser scanners were used to obtain strain and displacement profiles distributed around the structure. The soil-structure interaction problem was modelled with a cracked equivalent Timoshenko beam resting on coupled springs and subjected to greenfield tunnelling-induced forces at ground level. This model was investigated parametrically to better interpret the data. The results from the analyses agreed well with visual observations of damage and highlighted important response mechanisms which are not considered by conventional equivalent beam based damage assessment procedures. These response mechanisms relate to the transient settlements during intermediate stages of tunnel advance and the presence of pre-existing damage in the building. A modified equivalent beam procedure is proposed which accounts for both of these significant effects.
\end{abstract}

\section{Introduction}

The understanding of the impact of urban excavations on existing buildings is important for safe underground construction. Following the seminal work of Burland and Wroth (1974), engineers have derived modelling techniques which treat the building as an equivalent beam and subject it to tunnelling-induced greenfield displacements. In this approach, maximum strains due to greenfield displacements are calculated using beam theory and are then related to damage categories. More recently, design charts have been proposed to account for the significant influence of the relative soil and structural stiffnesses (e.g. soil structure interaction or SSI) on the displacements experienced by the structure (Potts and Addenbrooke, 1997). Alternatively, the soil-structure interaction can be modelled by numerical procedures. Amongst others, Franza and DeJong (2019) proposed a simple approach, which models the soil structure interaction problem with an equivalent beam resting on coupled springs.

Equivalent beam based assessment techniques have seen widespread use in major underground construction projects during the last decades (Mair et al., 1996). The simplicity of the assessment methods are ideally suited to the preliminary damage prediction of thousands of buildings affected by works during the planning stage. For these predictions, greenfield soil movements are directly imposed on the equivalent beams in order to calculate strains and damage categories. Furthermore, the same techniques are commonly used to evaluate the damage caused by tunnelling works during construction. In this approach, the real building displacement data, which is gathered by surveying equipment during construction, is used as an input for damage estimation.

The 'equivalent beam' modelling techniques are considered to be effective. However, they feature many simplifications which need to be evaluated. In particular, there is a need for detailed field measurements that can be used to evaluate the required complexity for the equivalent beam modelling approach to predict building displacements and strains due to underground construction.

To this end, this paper investigates the response of a façade of a Grade-1 listed masonry church affected by nearby tunnelling works. The project is introduced in Section 2, where standard equivalent beam assessment procedures are used to estimate the maximum strain experienced by the façade. In particular, two calculations are made. In the first calculation, the equivalent beam structure is subjected to greenfield displacements, estimated from empirical formulae using design volume losses. In the second calculation, structural deformations applied on the equivalent beam are taken from surveying data gathered during the project. Both assessment approaches are then compared to the detailed field data collected from fibre optic strain sensing and laser scan displacement monitoring techniques, revealing several 
Figure 1 (a) A drawing of the south façade of St Mary Abchurch, highlighting key aspects of geometry and instrumentation and (b) a plan view demonstrating the position of the building with respect to the tunnel, with two critical tunnel advances highlighted. (c-d) Schematics describing the parameters of the empirical 3D ground displacement model used in this study.

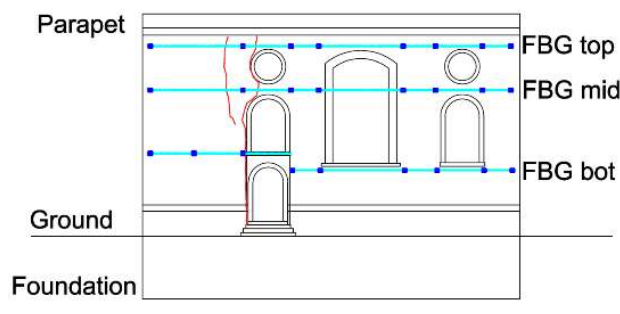

(a)

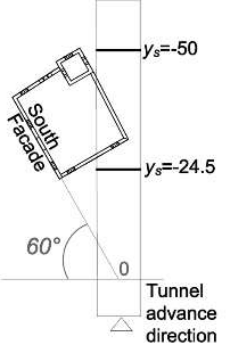

(b)

discrepancies. In Section 3, a modified equivalent beam modelling procedure is proposed and a parametric study is performed to investigate the mismatch between predicted and measured strains. In particular, numerical analyses of equivalent beam on coupled spring models are carried out, by accounting for transient settlement effects and existing cracks in the building. Section 5 summarises the results, highlighting important response mechanisms which need to be integrated into equivalent beam based damage assessment procedures.

\section{St Mary Abchurch and Bank Station Capacity Upgrade}

\subsection{Description of the structure and the project}

St Mary Abchurch is a Grade-I listed church in the Bank Conservation Area of the City of London. It was constructed following the Great Fire of London, by Christopher Wren. It has a roughly square plan, with four facades, each approximately $20 \mathrm{~m}$ long. The façades are constructed with $0.75 \mathrm{~m}$ thick double leaf brick with rubble fill. The doors, windows, quoins and various other ornamental structural elements are dressed in stone. A drawing of the south façade, which will be discussed in this paper, is shown in Figure 1a. It is noteworthy that the windows above the doorway highlight an existing branching crack (shown in red), which extends from the bottom of the doorway to the top of the structure. This crack is likely related to bomb damage during the second world war. The church is founded on a shallow lime concrete foundation resting on terrace gravels, $3.5 \mathrm{~m}$ below ground level.

Bank Station Capacity Upgrade project was designed to increase passenger safety and comfort in the Bank-Monument station complex. To achieve this, new running tunnels, escalator tunnels, cross-passages and a station complex is being constructed. The new Northern Line running tunnel passes underneath the northeastern corner of St Mary Abchurch (see Figure 1b). The tunnel was constructed using the New Austrian Tunnelling Method, $26.5 \mathrm{~m}$ under the foundations of the church, in the London Clay strata. The relevant construction works started in August 2017 and finished in January 2018.

\subsection{Building strains and damage using standard equivalent beam assessment procedures}

The equivalent beam method was used to estimate damage to the structure due to tunnelling-induced movements, following

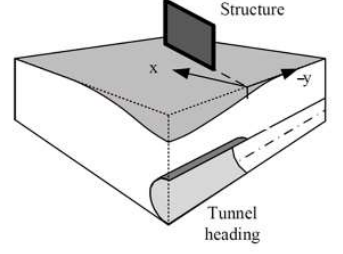

(c)

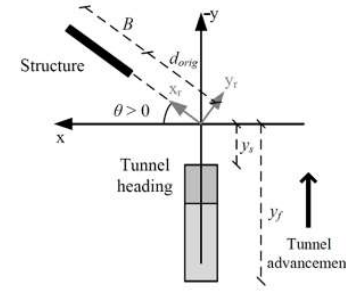

(d) the approach in Mair et al. (1996). To do this, vertical and horizontal greenfield movements at the foundations depth of the south façade were calculated using the 3D empirical formulas given by Camos and Molins (2015), which consider the building skew and tunnel advancement (see Figure $1 \mathrm{c}$ and d). A tunnel diameter of $9.8 \mathrm{~m}$, a design tunnel volume loss $V_{l, t}$ of $1.6 \%$ and trough width parameters $K_{x}$ and $K_{y}$ of 0.6 in the transverse and longitudinal directions were assumed. The angle of skew $\theta$ of 60 degrees and the eccentricity $d_{\text {orig }}$ of $24.4 \mathrm{~m}$ are defined.

For the equivalent beam calculations in this section, the tunnel portal and head were assumed to be outside the zone of the influence of the structure (corresponding to values of $y_{f}=50$

and $y_{s}=-50$ respectively, see Figure $1 \mathrm{c}$ and d). Therefore, in this section only the displacement field at the end of the tunnel construction is considered (i.e. the effects of transient settlement fields during tunnel advance were not considered). Due to the resulting settlements, the whole of south façade was located in the hogging zone. Finally, the relative deflection $\Delta$ was calculated from the vertical displacement field using standard procedures and normalised by the length of the façade $L$, which is $21 \mathrm{~m}$.

Table 1 Properties of the equivalent beam representing the south façade and the resulting strain values from the assessments

\begin{tabular}{ll}
\hline Properties & Value \\
Beam length, $L$ & $21 \mathrm{~m}$ \\
Beam height, $H$ & $15.5 \mathrm{~m}$ \\
Elasticity modulus, $E$ & $0.5 \mathrm{GPa}$ \\
Beam bending stiffness, $E I$ & $4.26 \mathrm{e} 10 \mathrm{Nm}^{2}$ \\
Beam axial stiffness, $E A_{a}$ & $4.34 \mathrm{e} 9 \mathrm{~N}$ \\
Beam shear stiffness, $G A_{s}$ & $6.58 \mathrm{e} 8 \mathrm{~N}$ \\
Maximum combined tensile strain & $40 \mu \varepsilon$ \\
$\varepsilon_{\max }$ calculated from greenfield & \\
displacement input & \\
Maximum combined tensile strain & $245 \mu \varepsilon$ \\
$\varepsilon_{\max }$ calculated from measured \\
building displacement input \\
\hline
\end{tabular}

The south façade of the church was characterised as a cross anisotropic Timoshenko beam. The properties of the beam 
Figure 2 (a) Vertical displacement measurements from levelling points and laser scanning along the length of the building façade, (b) all strain measurements from the façade versus time and (c) distribution of strain measurements along the façade at different heights.
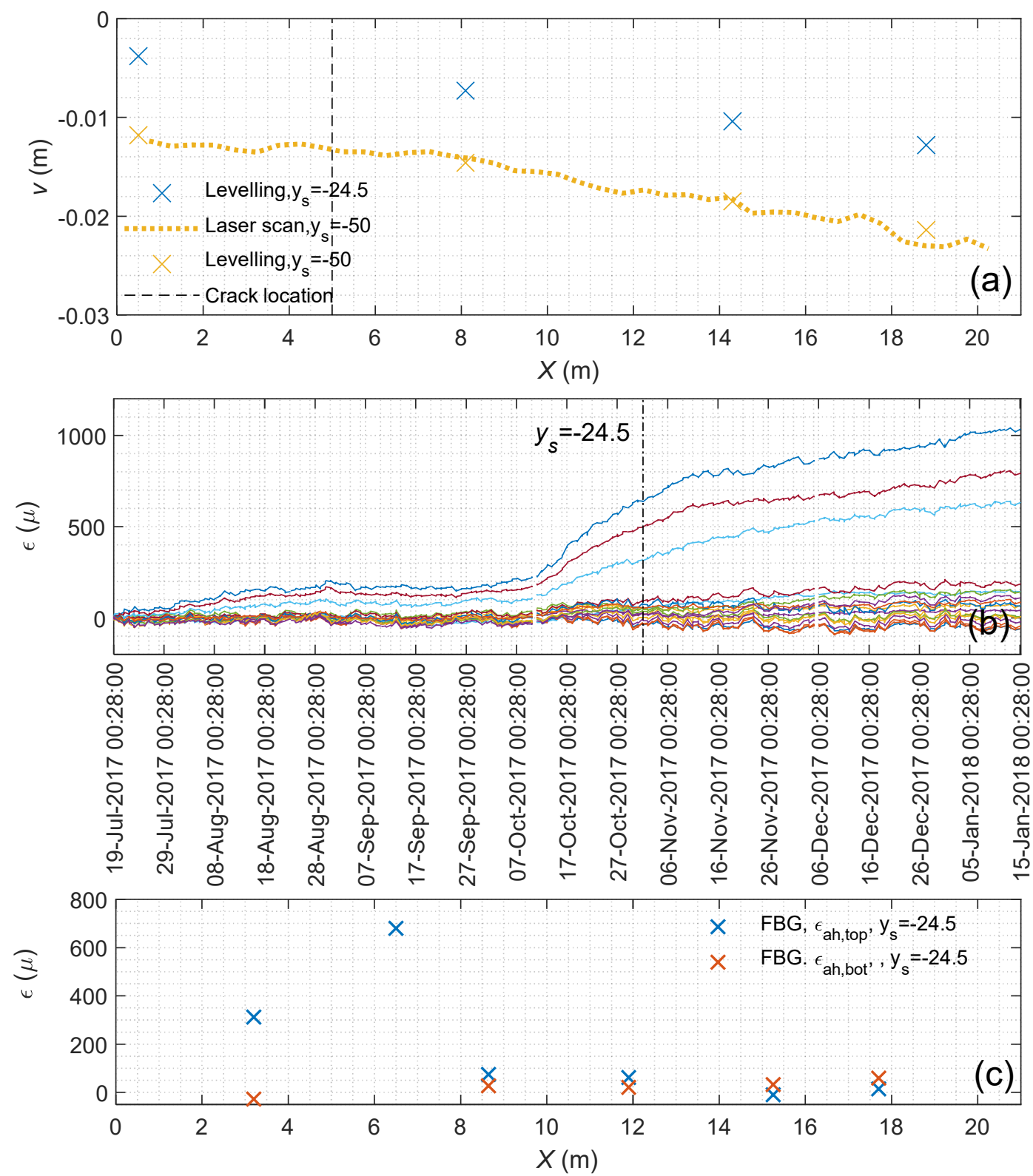

were calculated using the strip method (Pickhaver et al., 2010), results in a prediction of $40 \mu \varepsilon$, as listed in Table 1. This following the procedure discussed in Franza et al. (2019). Relevant dimensions and the reduced bending, shear and axial stiffness (denoted by $E I, G A_{s}$ and $E A_{A}$ ) are presented in Table 1. The maximum total tensile bending strain $\varepsilon_{b t, \text { max }}$ and the maximum total tensile strain due to diagonal distortion $\varepsilon_{d t, \max }$ are then calculated with the use of simple formulas defined by Mair et al (1996). The maximum combined tensile strain $\varepsilon_{\text {max }}$ is defined as the larger of $\varepsilon_{b t, \text { max }}$ and $\varepsilon_{d t, \text { max }}$.

Firstly, greenfield input displacements (horizontal and vertical movements) resulting from design values for ground losses were computed. These were imposed on the equivalent beam to estimate maximum combined tensile strain $\varepsilon_{\max }$. This corresponds to negligible (Category 0 ) damage. It is noteworthy that during construction, the real volume loss and the trough width parameters were calculated from greenfield data and these values agreed with the design values used herein for the equivalent beam analyses.

As an alternative approach, the input displacements for the equivalent beam assessment were estimated from monitoring data. The equivalent beam was therefore subjected to the horizontal and vertical displacements recorded at the south façade of the building. Figure $2 \mathrm{a}$ shows the vertical displacements of the south façade of the structure measured with levelling instruments at four different locations along the façade. Here, the abscissa $\mathrm{X}$ denotes the distance along the façade from the southwest corner. The diplacements are shown 
for two locations of tunnel advance, $y_{s}=-24.5$ and $y_{s}=-50$ (advancement of the heading is associated with decreasing $y_{s}$ ). The former shows the displacements when the tunnel is approximately underneath the northeastern façade of the building, while the latter describes the case where the tunnel has advanced beyond the zone of the influence of the facade (see Figure 1b). For $y_{s}=-50$, data points appear to lie on a straight line, with small relative displacements $\Delta$. Accompanying horizontal displacements, at the left and right corners of the façade and roughly at the mid-height of the structure, were measured from prisms as 3.9 and $7.7 \mathrm{~mm}$.

Applying these displacements to the equivalent beam, a maximum combined tensile strain $\varepsilon_{\max }$ of $245 \mu \varepsilon$ was obtained, as listed in Table 1. Interestingly, these estimated strains are notably higher than the predictions obtained from design greenfield movements, indicating an unexpected response. However, the estimated strains are still quite small and correspond to Category 0 damage.

Visual observations of damage in this façade broadly agree with these predictions and estimations. In the south façade, except for the widening of existing cracks around and above the doorway and sticking in the church doors, no visual signs of damage were observed.

\subsection{Comparison of equivalent beam prediction and assessments to distributed strain and displacement data}

Standard surveying equipment produces sparse displacement measurements. To complement this information, two alternative sensing techniques were utilised. The first technique compares periodically gathered laser scan point clouds to determine vertical displacements of the structure continuously along its length. To do this, the differences in elevation of the base parapet walls (highlighted in Figure 1a) were computed from the point clouds using the PAM algorithm (Acikgoz et al., 2017). The second sensing technique uses chains of Fibre Bragg Grating (FBG) sensors, which measure temperature compensated strains along the length of the façade, at three different heights over approximate gauge lengths of $3 \mathrm{~m}$ (see Figure 1a). To the authors' knowledge, this represents a first of its kind application of this technique for investigating tunnelling-induced damage.

Figure 2a demonstrates the vertical displacements calculated from laser scan point clouds (with a dotted line) for the case where the tunnel has advanced beyond the zone of influence of the facade $\left(y_{s}=-50\right)$. This agrees well with the data from the levelling points. In addition, the approximate location of the branching crack around and above the doorway is highlighted in this figure. Interestingly, the slope of the vertical displacement signal appears to change subtly in the region of the crack ( $\mathrm{X}=4$ to $6 \mathrm{~m}$ ). This particular feature of the response is not currently captured by the equivalent beam modelling techniques employed in Section 2.2.

Figure $2 \mathrm{~b}$ presents the strains measured by FBGs on the south façade with time. In this figure, it is possible to see the construction of the pilot tunnel between August and October
2017, and the following construction of the enlargement between November 2017 and January 2018. A significant increase of strain is observed as the tunnel is approaching the building. To highlight this, the location where the enlarged tunnel has advanced to just underneath the building ( $\left.y_{s}=-24.5\right)$ is shown with a vertical line. At this stage the measured strains reach approximately $60 \%$ of their final and maximum value. Positive strains indicate that tension was measured predominantly across the three lines of fibres located between mid-height and full height of the structure. It is important to note that out of the 18 FBGs, 15 indicate strain measurements with magnitudes less than $200 \mu \varepsilon$. This is in good agreement with the predictions and estimations made by the equivalent beam models. However, three FBGs, which are located around the doorway and traverse the crack, measure strain values exceeding $500 \mu \varepsilon$. This qualitatively agrees with the laser scan data which indicated a localised change in response around the location of the crack.

Figure $2 \mathrm{c}$ plots the strain distribution against the façade length along the top and bottom layer fibres for $y_{s}=-24.5$. This represents the case where the tunnel head is just underneath the northeastern corner of the building, and is chosen for later comparisons to data from equivalent modelling in Section 3. Readings of high strain correspond to locations where fibres measure over the existing branching crack around the doorway, which were sketched in Figure 1.

In general, Figure 2 demonstrated important discrepancies between the data and standard equivalent beam assessment procedures. In particular, the omission of the impact of existing damage resulted in unconservative predictions of strain. Equivalent beam representation of structures are modified and parametrically investigated in Section 3 to better capture structural response.

Figure 3 A schematic illustrating the numerical cracked equivalent beam models developed in this study.

(a)

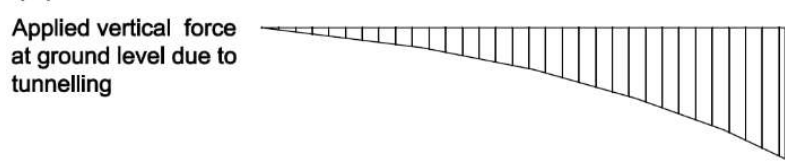

(b)
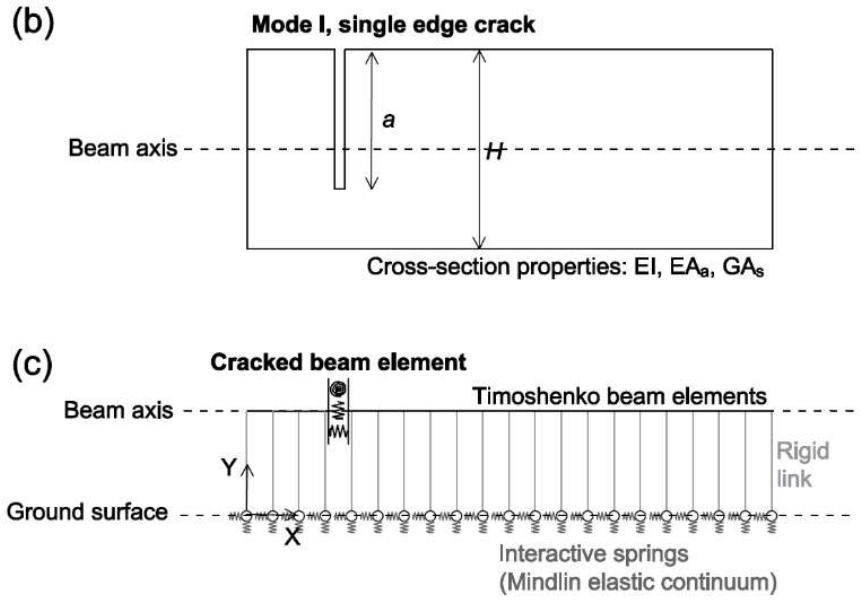
Figure 4 Analysis results for the uncracked equivalent beam model. (a) Horizontal and (b) vertical displacements experienced at the ground level during different stages of tunnel advance, and accompanying (c) total tensile bending strains at the extreme fibres of the beam. (d) Maximum combined tensile strains in the beam versus tunnel advance.
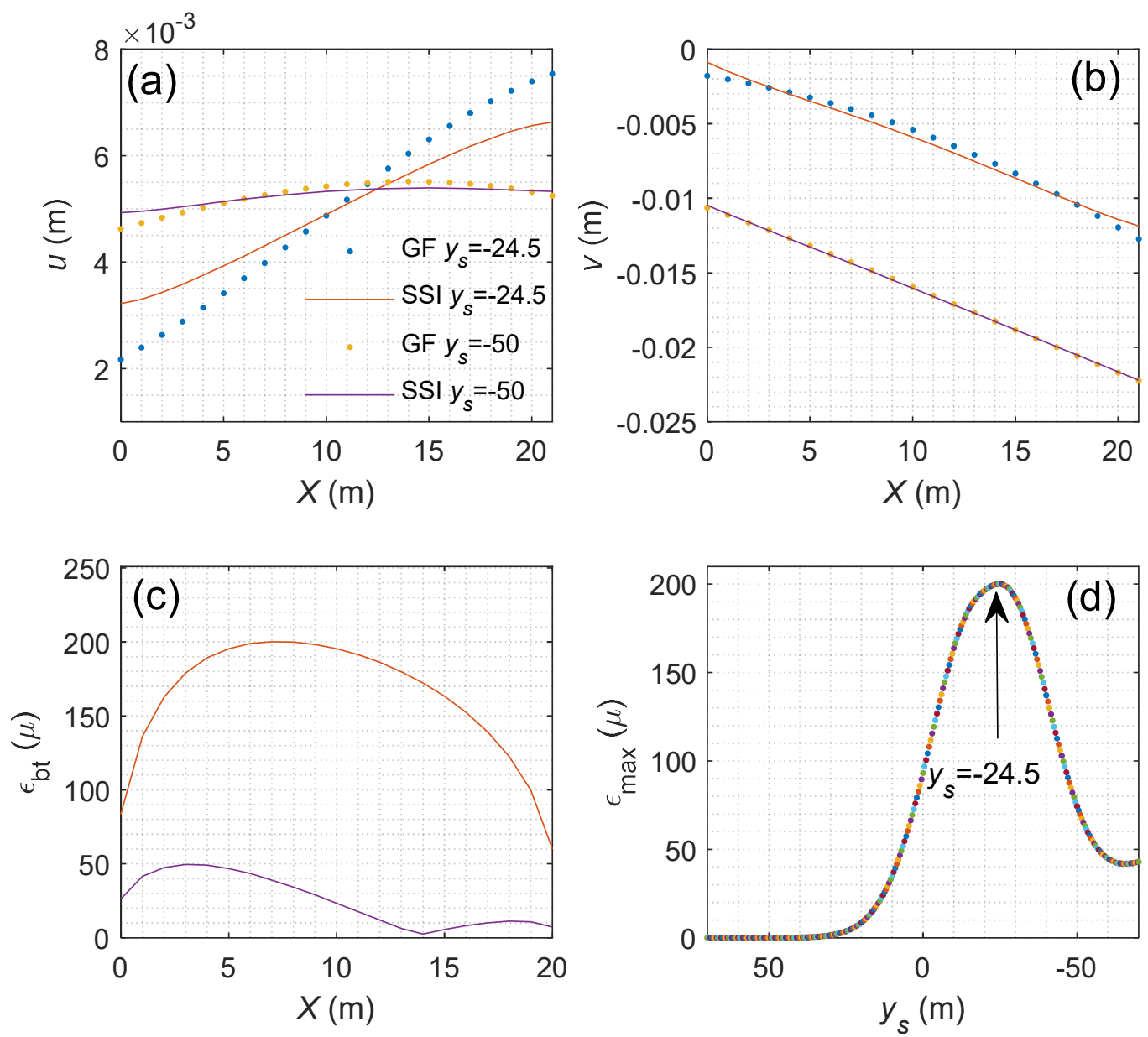

\section{Cracked equivalent beam models}

Recently, Franza and DeJong (2019) have proposed a modification of the standard numerical equivalent beam modelling technique discussed in the previous sections. As opposed to the standard equivalent beam approach where greenfield displacements are applied to the beam, Franza and DeJong's (2019) approach can be used to numerically simulate soil-structure interaction and estimate the displacement fields experienced by the structure.

In this approach the structure is represented as a EulerBernoulli beam placed on an elastic half-space representing the soil. By using integrated forms of Mindlin's equations, the stiffness of the elastic half-space at foundation level can be represented by a series of coupled horizontal and vertical springs. The stiffness matrix of the whole system is given by the sum of the individual contributions from the half-space and the structure. By subjecting this structure to tunnelling-induced greenfield forces at the ground level, the soil-structure interaction can be simulated. The direct calculation of strains

from the computed structural displacements is done by beam theory and is straightforward.

Subsequently, Franza et al. (2019) extended their approach to account for the shear deformability and neutral axis offset of the structure. This was achieved by idealising the structure as a cross anisotropic Timoshenko beam, located at its neutral axis and connected to soil nodes with rigid links. However, these previous works focused on the effects of steady-state ground movements obtained at the end of the tunnel excavation, and the beam was assumed orthogonal to the tunnel longitudinal axis.

In this paper, these equivalent beam on coupled spring models are further improved to investigate aspects that are typically neglected standard equivalent beam assessment procedures. First, to account for the influence of the transient displacements obtained as the tunnel is advancing, the 3D empirical method for greenfield displacements of Camos and Molins (2015) (discussed earlier in Figure 1c and d) was used. This model is used to calculate tunnelling-induced forces on the soilstructure interaction system for any tunnel advance (see Figure 3a for a representation of vertical forces applied on the nodes). 
Secondly, to account for the local flexibility of the structure at the location of the existing crack around the doorway, new cracked Timoshenko beam elements (location indicated in Figure $3 b$ and $c)$ were derived. This required modelling the varying flexibility of the beam along its length. Away from the crack, the flexibility is defined by classical cross-sectional properties of the beam. For instance, in this case, EI defines the bending stiffness. However, at the location of the crack, the flexibility is increased by a Dirac delta function, which assumes the value of a constant $\chi$ when the crack is opening. Following the approach of Dona et al. (2015), the constants $\chi_{b}$ , $\chi_{s}$ and $\chi_{a}$ are used to describe increases in bending, shear and axial flexibility (denoted by $1 / E I, 1 / E A_{a}$ and $1 / G A_{s}$ ) at the location of the crack.

To determine the local flexibility constants $\chi$, fundamental results from linear elastic fracture mechanics are used. Using the stress intensity factors of a plate of finite width and a single edge crack length $a$ (Broek, 1982: pp 76) and following the derivation of Anifantis and Dimarogonas (1983) and
Ostachowicz and Krawczuk (1991), the local flexibility constants for axial, bending and shear flexibilities can be determined for Mode-I or Mode-II cracks. Note that the additional flexibility due to the crack is only observed when tensile strains are observed at the crack centreline (e.g. when the crack is opening). Otherwise, under compression, the crack is assumed to be fully closed and the cross-sectional stiffness of the beam is assumed. This modelling approach, which assumes the crack as fully open or fully closed depending on the presence of tensile forces in the crack, is called a switching crack model (e.g. Dona et al., 2015)

For brevity, further details of the modelling approach are not presented. However, it is useful to note that this modelling approach is equivalent to modelling the structure as beams connected by rotational, shear and axial springs at the crack location (see Figure 3c). As such, it does not model crack progression or hysteretic behaviour (Dona et al., 2015), but could be refined to do so. Cracked beam elements have closedform stiffness matrices, with the same number of degrees of freedom at nodes as uncracked beam elements. This simple

Figure 5 Analysis results for the cracked equivalent beam model. (a) Horizontal and (b) vertical displacements at the ground level for cracked and uncracked beams, and accompanying (c) total tensile bending strains at the extreme fibres of the beam. (d) Maximum combined tensile strains in the undamaged part of the beam and averaged crack strains versus normalised crack length.
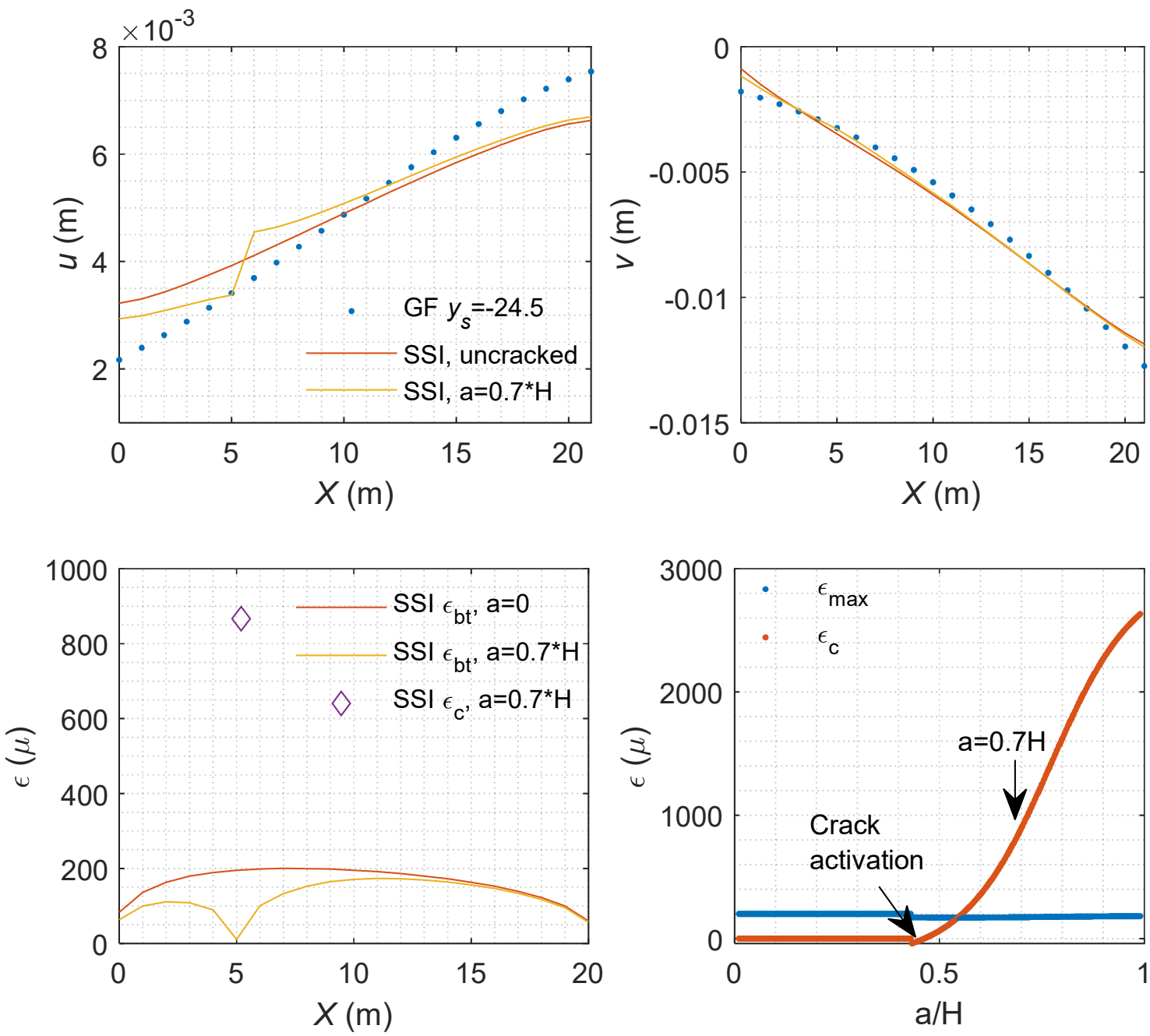
approach is well-suited for preliminary equivalent beam assessments, in order to understand the impact of existing damage on the soil structure interaction problem. In the following sections, the tunnelling-induced displacements and deformations of cracked equivalent beam models are explored parametrically to identify the key aspects that need to be modelled to better capture the structural response of St Mary Abchurch.

\subsection{Numerical models}

The properties of the cross-anisotropic Timoshenko beam and the empirical Greenfield parameters which were used for standard equivalent beam assessments were defined in Section 2. The same properties will be used in this section. Some additional parameters are required for describing the numerical models. These include $E_{s}$ and $v_{s}$, which respectively are the elastic modulus and Poisson's ratio of the soil. The former was defined as the elastic modulus of a soil sample taken at the middepth of the excavation, following the approach of Goh (2010).

\subsection{The influence of tunnel advance on response}

The southern façade of St Mary Abchurch has a skew angle of 60 degrees. Camos and Molins (2015) demonstrated that buildings with large skew angles may experience transient settlements during tunnel advance that cause strains larger than at the final steady-state condition. In fact, if the building remains elastic during the tunnel drive, tunnelling-induced strains may eventually reduce as the tunnel advances.

Camos and Molins' work (2015) did not explore the influence of soil-structure interaction, which plays an important role in the response of St Mary Abchurch. Using the modified equivalent beam on an elastic half-space, the influence of tunnel advance is explored in Figure 4. For this initial assessment, uncracked structures are considered.

Figure $4 \mathrm{a}$ and $\mathrm{b}$ respectively describe the ground horizontal and vertical displacements (denoted by $u$ and $v$ respectively in the direction of $\mathrm{X}$ and $\mathrm{Y}$ coordinates, see Figure 3). The filled circular markers describe the greenfield displacements (denoted as GF), while lines are used for the beam displacements arising from the soil-structure interaction analyses (denoted as SSI). In both figures, GF and SSI displacements are presented for two different advances, $y_{s}=-24.5$ and $y_{s}=-50$. From these figures, it is clear that $y_{s}=-24.5$ is associated with larger differential horizontal displacements and relative deflections on the structure. However, the soil structure interaction appears to change the governing response mechanisms and reduce the influence of these displacements on the structure. Nonetheless Figure 4c illustrates that there is considerable difference between the tensile total bending strains. Consistent with earlier work (Mair et al., 1997) $\varepsilon_{b t}$ is defined as the tensile strain experienced by one of the extreme fibres of the beam, calculated as the sum of bending and axial strains. The maximum total bending strain of $200 \mu \varepsilon$ experienced by the beam for $y_{s}=-24.5$ is approximately four times higher than the corresponding strain for $y_{s}=-50$. Similar results are obtained for diagonal tensile strain due to diagonal distortion $\varepsilon_{d t}$, but these are not discussed as the strain magnitudes are lower. The findings are further illustrated in Figure 4d where the maximum combined tensile strain $\varepsilon_{\max }$ experienced by the beam is plotted against tunnel advance, highlighting that the strains are largest when the tunnel is underneath the building at $y_{s}=-24.5$. However, it is noteworthy that advance effects are not sufficient to explain the field strain data, where significantly higher strains were measured.

\subsection{The influence of pre-existing cracks on response}

Figure $5 \mathrm{a}$ and $\mathrm{b}$ plot the horizontal and vertical displacements at the ground level of uncracked and cracked equivalent beams for the critical advance, i.e. $y_{s}=-24.5$. Existing damage is modelled as a Mode I crack, which influences only the axial and bending stiffness of the structure. The single edge crack was located at $\mathrm{X}=5.2 \mathrm{~m}$ with the vertical length $a$ of $0.7 \mathrm{H}$, which corresponds to the vertical distance of the bottom of the doorway to the top of the structure (see Figure 1). In Figure 5a, the influence of the crack can be clearly observed; the horizontal displacements to the left and right side of the crack are significantly different. The vertical displacement traces in Figure $5 \mathrm{~b}$ are subtly different. On closer inspection, it is possible to see that the vertical displacement of the cracked structure is flatter in the uncracked sections, and there is a kink at the crack location. This aspect of response mirrors the behaviour observed in the laser scan data, discussed in Figure $2 \mathrm{a}$.

In Figure 5c, total bending strains $\varepsilon_{b t}$ are plotted. For the cracked structure, the strain distribution in the undamaged parts are plotted alongside an averaged crack strain $\varepsilon_{c}$. The averaged crack strain is estimated by calculating the total bending strain at the top of the beam over a distance of $3 \mathrm{~m}$ with the crack location in the middle. The presence of the crack causes a redistribution of strains, although strain values in the uncracked parts of the beam are always less than $200 \mu \varepsilon$. There is an averaged crack strain of $800 \mu \varepsilon$, due to the relative rotation between left and right sides of the crack (see the horizontal displacements around the cracked beam element in Figure 5a). It should be noted that this corresponds to a crack opening of $\approx 2.4 \mathrm{~mm}$ if all strain is assumed to be concentrated in the crack. The current averaging over $3 \mathrm{~m}$ was selected to facilitate comparison to FBG measurements in Figure 2c. It can be observed that the real measurements indicate a branched crack, the effect of which can be seen over two FBGs located near the doorway, with approximate values of 300 and $700 \mu \varepsilon$

. The simulated value of $800 \mu \varepsilon$ captures the localisation of strains in the same area, and indicates good overall agreement.

Figure $5 \mathrm{~d}$ explores the averaged crack strain, $\varepsilon_{c}$, and the maximum combined tensile strain in the undamaged part of the beam, $\varepsilon_{\text {max }}$, for different crack lengths. All crack lengths refer to the single edged crack shown in Figure $3 \mathrm{~b}$, where the crack initiates from the top of the structure and extends to a height $a$. For the given scenario, crack opening only starts for a crack length of $a=0.45 H$. For smaller sizes, the crack does not open 
and the flexibility of the beam is not reduced, resulting in identical response for all beams up to this length of crack. In the uncracked part of the beam maximum strains get marginally smaller as the crack length increases. However, the increase in crack strains is much more significant and needs to be taken into account for damage assessments.

\section{Conclusions}

This paper presented new insights gathered from monitoring data and equivalent beam modelling of a church façade affected by tunnelling-induced displacements. The façade is positioned at a large skew angle with respect to the tunnel. The monitoring data indicated a quick build-up of strains during early stages of construction as the tunnel approached the building. A rotational discontinuity was observed in the displacement trace and significant tensile strains were measured around the crack. Strains measured elsewhere in the façade were small.

Standard equivalent beam assessment procedures were then used to evaluate strains experienced in St Mary Abchurch. First, predictions of strains induced by design greenfield movements at the end of the tunnel construction were evaluated. Then, the equivalent beam model was subjected to the displacements the façade measured in the field. Both assessments suggested that negligible strains in the façade, which did not agree with the field data.

To understand the reasons for the discrepancies, analyses were carried out on equivalent beam models resting on an elastic continuum and subjected to tunnelling-induced forces. These numerical models were modified to capture the influence of transient settlements during tunnel advance on the structural response. In line with earlier studies, it was determined that facades with a large skew angle may experience the largest strains during earlier stages of tunnel drive, as the tunnel is approaching the building.

To account for the influence of pre-existing damage on the soilstructure interaction problem, a simplified beam finite element with concentrated damage was adopted. This model simulates the macroscopic effects of damage with Dirac's delta functions in the flexibility functions of the beam element. It is equivalent to elastic rotational, shear and axial springs at crack locations, the properties of which depend on crack size and are defined using classical solutions of fracture mechanics.

The inclusion of a beam element with a Mode I switching edge crack in the model drastically altered the soil-structure interaction problem for large crack lengths. For small crack lengths, the crack remained closed, and no differences were observed. However, modelling the large crack in St Mary Abchurch resulted in simulations which effectively capture the behaviour observed by the distributed displacement and strain data. The results emphasise that large strains are observed at the crack location, while marginally reducing the strains elsewhere in the structure.

On the basis of these results, it is recommended to account for transient settlement effects during tunnel advance and pre- existing structural damage during equivalent beam analyses and risk assessments. If these models indicate potential for significant straining at the crack location, then, it may be necessary to conduct more detailed finite element models which model crack propagation. Finally, it is noted that without the detailed field data from fibre optic sensors and laser scanners, these developments in assessment procedures would not have been achieved. Where possible, such detailed data should be gathered during underground construction projects to further improve assessment procedures.

\section{Acknowledgements}

The authors would like to acknowledge the contribution of the following in installing, gathering and processing of the field data in Figure 2: Jason Shardelow, Cedric Kechavarzi, Paul Fidler and James Douie from the University of Cambridge, Marco Nunzio Pascariello, Antonio Luciano and Emilio Bilotta from the University of Naples Federico II, Mark Dewhirst and Keith Bowers from Transport for London, Antonio Santos and Julian Portillo-Aguilar from Geocisa and the organisation Friends of the City Churches.

\section{References}

Acikgoz S et al. (2017). Evaluation of the response of a vaulted masonry structure to differential settlements using point cloud data and limit analyses. Construction and Building Materials, 150: 916-931.

Anifantis N \& Dimarogonas A (1983). Stability of columns with a single crack subjected to follower and vertical loads. International Journal of Solids and Structures, 19(4): 281-291.

Broek D (1982). Elementary engineering fracture mechanics. Martinus Nijhoff Publishers, The Hague, Netherlands, pp.76.

Burland JB \& Wroth CP (1974). Settlement of buildings and associated damage. Conference on Settlement of Structures, Cambridge, UK.

Camós C \& Molins C (2015). 3D analytical prediction of building damage due to ground subsidence produced by tunneling. Tunnelling and Underground Space Technology 50: 424-437.

Donà $\mathrm{M}$ et al. (2015). An efficient two-node finite element formulation of multi-damaged beams including shear deformation and rotatory inertia. Computers \& Structures 147: 96-106.

Franza A \& DeJong, MJ (2019). Elastoplastic solutions to predict tunnelling-induced load redistribution and deformation of surface structures. Journal of Geotechnical and Geoenvironmental Engineering 145(4).

Franza A et al. (2019). Timoshenko beam models for the coupled analysis of building response to tunnelling. Tunnelling and Underground Space Technology. Under review. 
Goh KH (2011). Response of ground and buildings to deep excavations and tunnelling. $\mathrm{PhD}$ thesis, University of Cambridge, Cambridge, UK.

Mair RJ et al. (1996). Prediction of ground movements and assessment of risk of building damage due to bored tunnelling. Proceedings of the Geotechnical Aspects of Underground Construction in Soft Ground, London, UK.

Ostachowicz WM \& Krawczuk M (1991). Analysis of the effect of cracks on the natural frequencies of a cantilever beam. Journal of Sound and Vibration 150(2): 191-201.

Potts DM \&. Addenbrooke TI (1997). A structure's influence on tunnelling-induced ground movements. Proceedings of the ICE - Geotechnical Engineering 125(2): 109-125.

Pickhaver JA et al. (2010). An equivalent beam method to model masonry buildings in 3D finite element analysis. Computers \& Structures 88:1049-1063. 